\title{
Constraints on the annihilation cross section of dark matter particles from anisotropies in the diffuse gamma-ray background measured with Fermi-LAT
}

\author{
Shin'ichiro Ando \\ Gravitation Astroparticle Physics Amsterdam (GRAPPA) and Institute for Theoretical Physics, \\ University of Amsterdam, 1090 GL Amsterdam, The Netherlands \\ Eiichiro Komatsu \\ Max-Planck-Institut für Astrophysik, Karl-Schwarzschild Str. 1, 85741 Garching, Germany \\ Kavli Institute for the Physics and Mathematics of the Universe, \\ Todai Institutes for Advanced Study, the University of Tokyo, \\ Kashiwa, Japan 277-8583 (Kavli IPMU, WPI) and \\ Texas Cosmology Center and the Department of Astronomy, \\ The University of Texas at Austin, 1 University Station, C1400, Austin, TX 78712, USA
}

(Dated: January 24, 2013)

\begin{abstract}
Annihilation of dark matter particles in cosmological halos (including a halo of the Milky Way) contributes to the diffuse gamma-ray background (DGRB). As this contribution will appear anisotropic in the sky, one can use the angular power spectrum of anisotropies in DGRB to constrain properties of dark matter particles. By comparing the updated analytic model of the angular power spectrum of DGRB from dark matter annihilation with the power spectrum recently measured from the 22-month data of Fermi Large Area Telescope (LAT), we place upper limits on the annihilation cross section of dark matter particles as a function of dark matter masses. We find that the current data exclude $\langle\sigma v\rangle \gtrsim 10^{-25} \mathrm{~cm}^{3} \mathrm{~s}^{-1}$ for annihilation into $b \bar{b}$ at the dark matter mass of $10 \mathrm{GeV}$, which is a factor of three times larger than the canonical cross section. The limits are weaker for larger dark matter masses. The limits can be improved further with more Fermi-LAT data as well as by using the power spectrum at lower multipoles $(\ell \lesssim 150)$, which are currently not used due to a potential Galactic foreground contamination.
\end{abstract}

PACS numbers: 95.35.+d, 95.85.Pw, 98.70.Vc

\section{INTRODUCTION}

Understanding the identity and nature of dark matter, which makes up more than $80 \%$ of the total matter density in the Universe, is a major goal of modern physics and cosmology. The most promising candidate for dark matter is the weakly interacting massive particles (WIMPs), with which one can naturally explain the observed dark matter density using a simple thermal freeze-out argument 11. If dark matter particles annihilate into standard model particles, as expected for most WIMP scenarios, one can indirectly detect and constrain properties of dark matter particles [1 $[3$. In this paper, we shall focus on high-energy (1-50 GeV) gamma-ray photons produced by the cascade of annihilation products.

Dark matter annihilation occurs in all cosmological halos including a halo of the Milky Way, and thus contributes to the diffuse gamma-ray background (DGRB) 4 8. Due to the large-scale structure of the Universe, the observed gamma-ray emission appears anisotropic in the sky in a predictable manner, which makes it easy to identify the dark matter origin of highenergy gamma rays in the sky [9] (also see Refs. [10 26]).

Recently, the Fermi-LAT collaboration has measured the power spectrum of DGRB anisotropy from the 22month of data 27. They have detected significant excesses of the angular power spectrum over the shot noise of photons for a multipole range between $\ell=155$ and 504 and for multiple energy bins. A further study shows that most of these excesses come from unresolved blazars 28 . Subtracting the estimate of the blazar contribution, we have upper bounds on the residual anisotropy of DGRB.

In this paper, we use the upper bounds on the power spectrum to constrain the annihilation cross section of dark matter particles. For this purpose, we update our theoretical framework for computing the angular power spectrum presented in Refs. [9, 10, 20, as follows:

1. We use the results from recent numerical simulations (e.g., Ref. 29]) to model the mass function and spatial distribution of subhalos within a given host halo.

2. We include contributions from both the extragalactic dark matter halos and the Galactic dark matter subhalos.

3. We also include the cross correlation between dark matter annihilation signals and blazars. Although this term was often ignored in the literature (except for Ref. [10]), one should include this term for selfconsistency: the same halo hosting a blazar also contains annihilating dark matter particles, and there is a spatial correlation between halos hosting blazars and those not hosting blazars but containing annihilating dark matter particles. 
This paper is organized as follows. In Sec. III we present the predicted angular power spectrum of DGRB from extragalactic dark matter halos, and compare this to the data to find constraints on the annihilation cross section. In Sec. III, we discuss the contribution from the cross-correlation term with blazars. In Sec. IV] we present the predicted angular power spectrum of DGRB from Galactic subhalos, and find combined constraints on the annihilation cross section using extragalactic and Galactic contributions. We conclude in Section $\mathrm{V}$. Throughout the paper, we adopt a flat cold dark matter model with a cosmological constant $(\Lambda \mathrm{CDM})$ with the following cosmological parameters: $\Omega_{m}=0.277$, $\Omega_{\Lambda}=0.723, H_{0}=100 h \mathrm{~km} \mathrm{~s}^{-1} \mathrm{Mpc}^{-1}$ with $h=0.7$, $n_{s}=0.96$, and $\sigma_{8}=0.81$.

\section{EXTRAGALACTIC CONTRIBUTION}

In this section, we discuss the mean intensity and anisotropy of DGRB from dark matter annihilation in extragalactic halos. Much of the calculations are based on our earlier work [9, 10, but with extensions of the framework and significant updates on input models as explained below.

\section{A. Mean intensity}

The mean intensity of gamma rays from dark matter annihilation is given by ${ }^{1}$

$$
I(E)=\int d \chi W([1+z] E, \chi)\left\langle\delta^{2}\right\rangle,
$$

where $E$ is the energy of photons, $\chi$ is the comoving distance to a source at redshift $z[\chi$ and $z$ are used interchangeably through the relation, $d \chi / d z=c / H(z)],\left\langle\delta^{2}\right\rangle$ is the variance of the overdensity field, $\delta=(\rho-\langle\rho\rangle) /\langle\rho\rangle$, and is often also referred to as an "intensity multiplier." $W(E, z)$ is the window function that contains particlephysics information such as a velocity-averaged annihilation cross section times relative velocity, $\langle\sigma v\rangle$, a dark matter mass, $m_{\mathrm{dm}}$, and a gamma-ray spectrum per annihilation, $d N_{\gamma, \text { ann }} / d E$ :

$$
W(E, z)=\frac{\langle\sigma v\rangle}{8 \pi}\left(\frac{\Omega_{\mathrm{dm}} \rho_{c}}{m_{\mathrm{dm}}}\right)^{2}(1+z)^{3} \frac{d N_{\gamma, \mathrm{ann}}}{d E} e^{-\tau(E, z)},
$$

where $\Omega_{\mathrm{dm}}=0.23$ is the density parameter of dark matter, and $\rho_{c}$ is the critical density of the present Universe. Here, $\tau(E, z)$ is the optical depth for a gamma ray emitted at energy $E$, for which we adopt the model

\footnotetext{
${ }^{1}$ Here we define the mean intensity, $I$, as the number of photons received per unit area, unit time, unit energy range, and unit solid angle, i.e., $I(E)=d N /(d A d t d E d \Omega)$.
}

of Ref. [30]. Note that the annihilation cross section required to produce dark matter at the right relic density by the thermal freeze-out mechanism with S-wave annihilation is $\langle\sigma v\rangle \simeq 3 \times 10^{-26} \mathrm{~cm}^{3} \mathrm{~s}^{-1}$, which is largely independent of the dark matter mass [1].

The intensity multiplier is

$$
\begin{aligned}
\left\langle\delta^{2}\right\rangle= & \left(\frac{1}{\Omega_{m} \rho_{c}}\right)^{2} \int d M \frac{d n(M, z)}{d M}\left[1+b_{\mathrm{sh}}(M)\right] \\
& \times \int d V \rho_{\text {host }}^{2}(r \mid M),
\end{aligned}
$$

where $M$ is the virial mass, $d n / d M$ is the halo mass function, for which we adopt an ellipsoidal collapse model [31, 32], $\rho_{\text {host }}(r \mid M)$ is the density profile of a host halo of mass $M, r$ is the comoving radius from the halo center, and $b_{\mathrm{sh}}(M)$ is a boost factor due to annihilation in subhalos.

We adopt an Navarro-Frenk-White (NFW) 33 profile for host halos,

$$
\rho_{\text {host }}(r)=\frac{\rho_{s}}{\left(r / r_{s}\right)\left(r / r_{s}+1\right)^{2}},
$$

where $\rho_{s}$ and $r_{s}$ are the scale density and the scale radius, respectively, and this relation holds out to the virial radius, $r_{\text {vir }}$, which, in turn, is given as a function of $M$ and $z$ through the relation: $M=4 \pi r_{\text {vir }}^{3} \Delta_{\text {vir }}(z) \rho_{c}(z) / 3$, with $\Delta_{\mathrm{vir}}(z)=18 \pi^{2}+82 d-39 d^{2}$ and $d=\Omega_{m}(1+z)^{3} /\left[\Omega_{m}(1+\right.$ $\left.z)^{3}+\Omega_{\Lambda}\right]-1$ [34.

The scale radius is defined as $r_{s}=r_{\text {vir }} / c_{\text {vir }}$, where $c_{\mathrm{vir}}(M, z)$ is the concentration parameter, for which we adopt the model of Ref. 35] for masses below $2.5 \times$ $10^{14} M_{\odot}$ and that of Ref. [36] otherwise. By taking the volume integral of the density profile, $\rho_{\text {host }}(r)$, out to $r_{\text {vir }}$ and equating it to $M$, we obtain the scale density as

$$
\rho_{s}=\frac{M}{4 \pi r_{s}^{3}}\left[\ln \left(1+c_{\mathrm{vir}}\right)-\frac{c_{\mathrm{vir}}}{1+c_{\mathrm{vir}}}\right]^{-1} .
$$

The volume integral of the density squared has an analytic form:

$$
\int d V \rho_{\text {host }}^{2}=\frac{4 \pi r_{s}^{3} \rho_{s}^{2}}{3}\left[1-\frac{1}{\left(1+c_{\mathrm{vir}}\right)^{3}}\right] .
$$

The gamma-ray intensity is further boosted by annihilation in subhalos, which is represented by the boost factor, $b_{\mathrm{sh}}(M)$, for which we adopt a fitting formula based on results of recent numerical simulations [29]: $b_{\mathrm{sh}} \approx 110\left(M_{200} / 10^{12} M_{\odot}\right)^{0.39}$, where $M_{200}$ is an enclosed mass within a radius $r_{200}$ in which the average density is 200 times the critical density; there is a simple relation between $M_{200}$ and the virial mass $M$ [37. This boost is realized if the subhalo mass function extends down to Earth-mass scales, $M_{\mathrm{sh}, \min }=10^{-6} M_{\odot}$, which is a typical cutoff scale for the neutralino dark matter [38,41]. We note, however, that the boost factor strongly depends on the minimum subhalo mass chosen, $b_{\mathrm{sh}} \propto M_{\mathrm{sh}, \min }^{-0.2}$ [29. Given that a wide range of minimum subhalo mass is still 


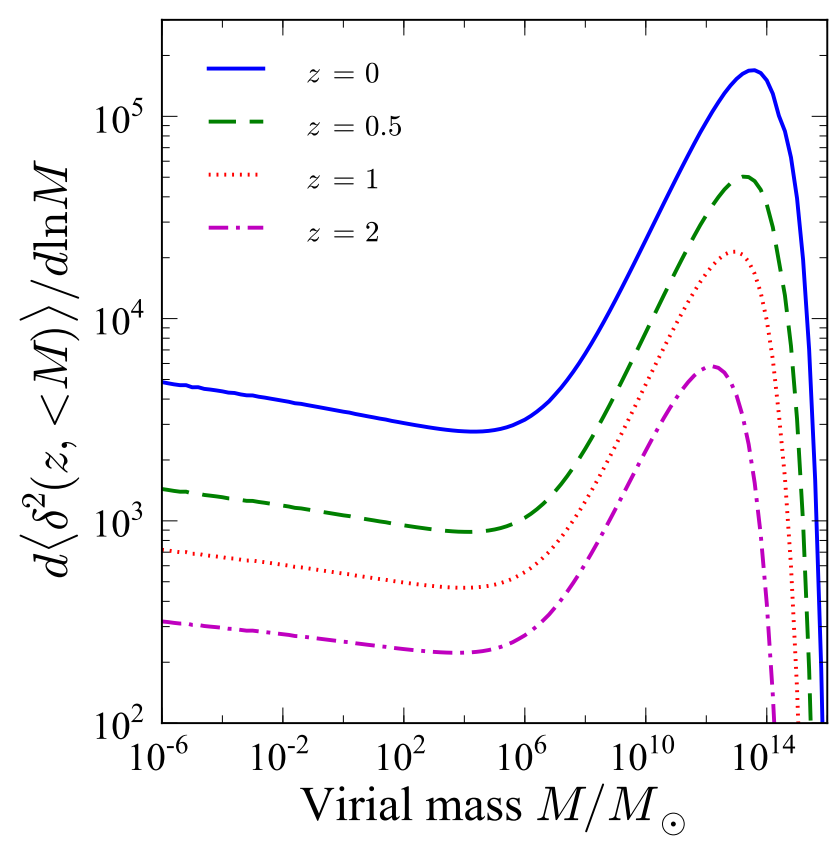

FIG. 1. Contribution to the intensity multiplier, $\left\langle\delta^{2}\right\rangle$ [Eq. [3]], from different mass ranges at various redshifts.

allowed, as small as $\sim 10^{-12} M_{\odot}$ 42, the annihilation rate may be boosted even further.

Figure 1 shows the integrand of Eq. (3) as a function of the virial mass, $M$, for various redshifts, $z$. Namely, this shows what fraction of $\left\langle\delta^{2}\right\rangle$ is contributed from which mass ranges. As can be seen from this figure, the dominant contribution comes from cluster-size ha$\operatorname{los}\left(M \sim 10^{14} M_{\odot}\right)$ at $z \sim 0$ and galaxy-size halos $\left(M \sim 10^{12} M_{\odot}\right)$ at $z \sim 2$. This is mainly because of the boost due to annihilation in subhalos. In Fig. 2, we compare the contribution from the host halos (dashed line) and that from the host halos and subhalos (solid line) at $z=0$. If there are no gamma ray from subhalos, then the mean intensity would be dominated by the smallest dark matter halos.

Figure 3 shows the intensity multiplier $\left\langle\delta^{2}\right\rangle$ as a function of redshifts for the case with and without subhalo contributions. Here we multiply $\left\langle\delta^{2}\right\rangle$ by $(1+$ $z)^{3} / \sqrt{\Omega_{m}(1+z)^{3}+\Omega_{\Lambda}}$ to show contributions to the mean intensity from different redshift ranges. One can see that the presence of subhalos boosts the intensity by a factor of 10 at low redshifts, and by a factor of $\sim 2$ even at $z=5$. The dotted lines are further multiplied by the absorption factor, $e^{-\tau}$, for observed energies of $E=10$, 20, 50, and $100 \mathrm{GeV}$. There is little absorption for photons received below $10 \mathrm{GeV}$, but this effect is significant for energies above tens of $\mathrm{GeV}$ and should be taken into account.

Figure 4 shows the predicted mean intensity of DGRB from dark matter annihilation with $m_{\mathrm{dm}}=100 \mathrm{GeV}$, $\langle\sigma v\rangle=3 \times 10^{-26} \mathrm{~cm}^{3} \mathrm{~s}^{-1}$, and the $b \bar{b}$ annihilation chan-

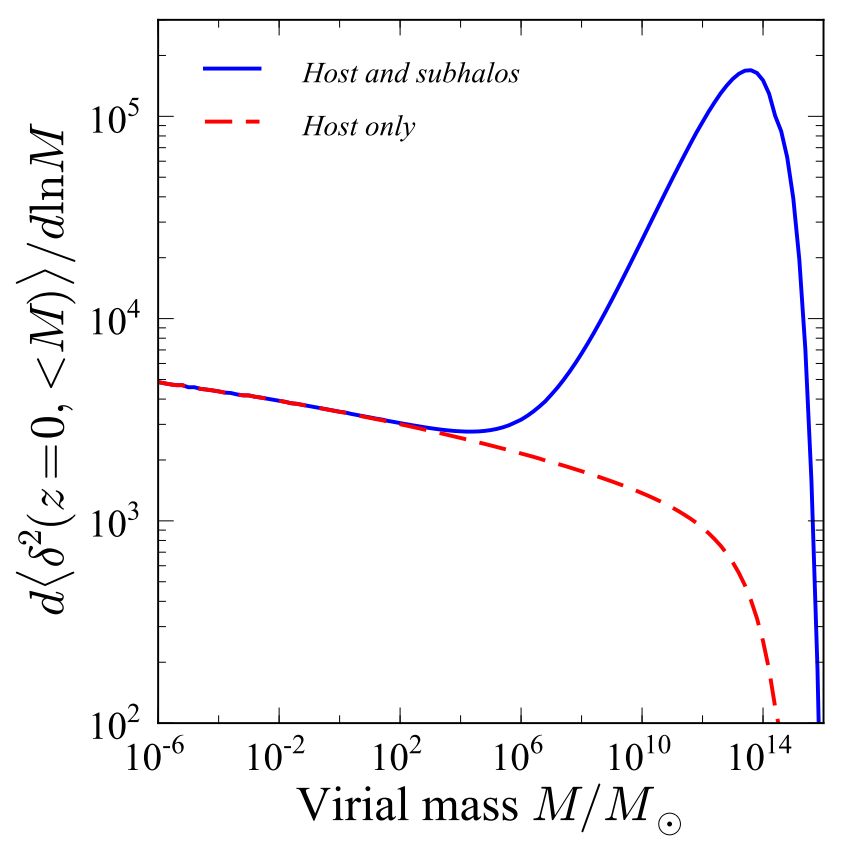

FIG. 2. Contributions to the intensity multiplier, $\left\langle\delta^{2}\right\rangle$ [Eq. (3)], from host halos (dashed) and host halos and subhalos (solid) at $z=0$. The solid line is the same as that in Fig. 1 .

nel. This model gives the dark matter contribution that is as large as $10 \%$ of the mean intensity measured by Fermi-LAT [43] at $E \sim 10 \mathrm{GeV}$. This contribution is quite significant given that even the most dominant contributors known to date, i.e., unresolved blazars, contribute to DGRB at around the same level [44].

\section{B. Angular power spectrum}

The angular power spectrum at a given multipole, $\ell$, is given by

$$
C_{\ell}(E)=\int \frac{d \chi}{\chi^{2}} W^{2}([1+z] E, z) P_{\delta^{2}}\left(k=\frac{\ell}{\chi}, z\right) .
$$

Following conventions of recent publications (e.g., Ref. 27]), our definition of the angular power spectrum [Eq. [7] has the units of $\left(\mathrm{cm}^{-2} \mathrm{~s}^{-1} \mathrm{sr}^{-1} \mathrm{GeV}^{-1}\right)^{2} \mathrm{sr}{ }^{2}$ which is referred to as the intensity angular power spectrum. In order to obtain the fluctuation angular power

\footnotetext{
${ }^{2}$ When we compare theoretical predictions with the data, we must integrate a gamma-ray intensity in a given direction over energy within a given energy bin. We do this by replacing the window function, $W([1+z] E, z)$, in Eqs. (1) and (7) with the window function integrated over a given energy range. This gives $C_{\ell}$ in units of $\left(\mathrm{cm}^{-2} \mathrm{~s}^{-1} \mathrm{sr}^{-1}\right)^{2} \mathrm{sr}$.
} 


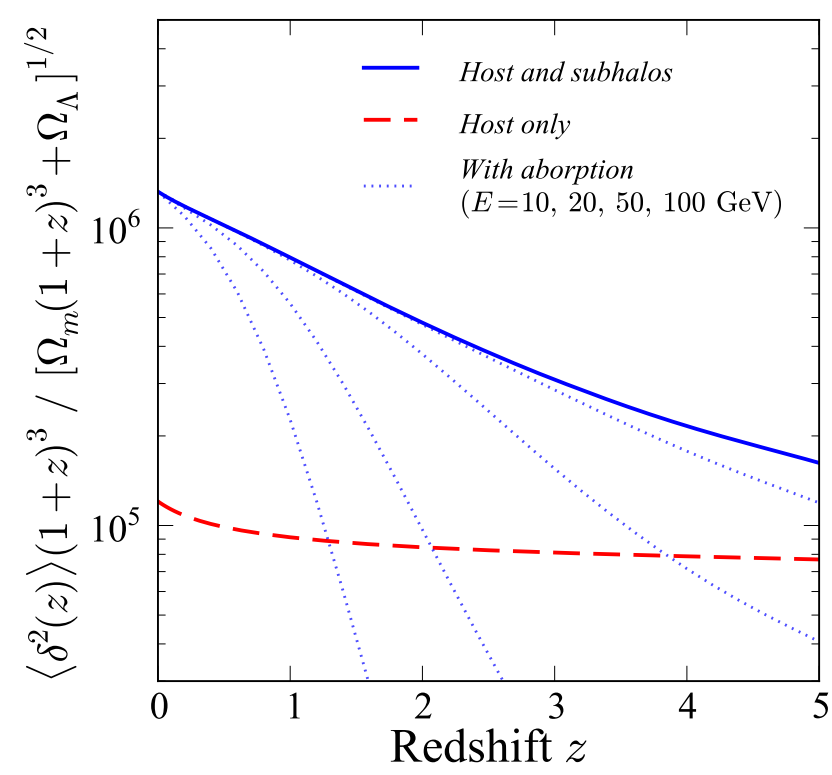

FIG. 3. Intensity multiplier as a function of redshifts. The solid line shows the total intensity multiplier from host halos and subhalos, while the dashed line shows the intensity multiplier only from host halos. The dotted lines show the total intensity multiplier multiplied by the absorption factors at various energies $(E=100,50,20$, and $10 \mathrm{GeV}$ from left to right).

spectrum that has units of sr and is adopted in the earlier papers (e.g., Ref. 9]), one simply divides the intensity power spectrum by the mean-intensity squared, $I^{2}(E)$.

Here, $P_{\delta^{2}}(k, z)$ is the power spectrum of the overdensity squared, $\delta^{2}$, which can be divided into one- and twohalo terms 9]:

$$
P_{\delta^{2}}(k, z)=P_{\delta^{2}}^{1 \mathrm{~h}}(k, z)+P_{\delta^{2}}^{2 \mathrm{~h}}(k, z) .
$$

The one-halo term correlates two points in one identical halo, whereas the two-halo term does that in two distinct halos. Correspondingly, the angular power spectrum is also divided into two terms:

$$
C_{\ell}(E)=C_{\ell}^{1 \mathrm{~h}}(E)+C_{\ell}^{2 h}(E)
$$

These two terms of the power spectrum can be explicitly

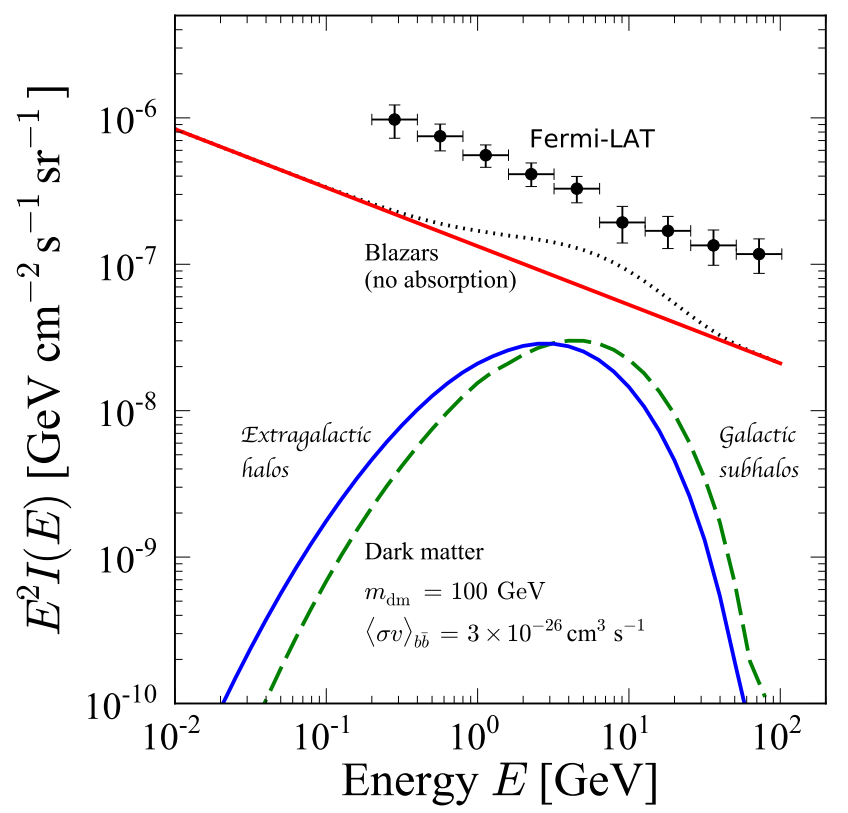

FIG. 4. Predicted mean intensity of DGRB from dark matter annihilation via the $b \bar{b}$ channel. The dark matter mass is $m_{\mathrm{dm}}=100 \mathrm{GeV}$, and the cross section is at its canonical value, $\langle\sigma v\rangle=3 \times 10^{-26} \mathrm{~cm}^{3} \mathrm{~s}^{-1}$. The solid line shows the extragalactic contribution, while the dashed line shows the Galactic subhalo contribution (no smooth Galactic contribution is included). The Fermi-LAT data [43] as well as the contribution from unresolved blazars are also shown for comparison.

written as

$$
\begin{aligned}
P_{\delta^{2}}^{1 \mathrm{~h}}(k, z)= & \left(\frac{1}{\Omega_{m} \rho_{c}}\right)^{4} \int d M \frac{d n(M, z)}{d M}|\tilde{u}(k \mid M)|^{2} \\
& \times\left[\left(1+b_{\mathrm{sh}}(M)\right) \int d V \rho_{\text {host }}^{2}(r \mid M)\right]^{2}, \quad(10) \\
P_{\delta^{2}}^{2 \mathrm{~h}}(k, z)= & {\left[\left(\frac{1}{\Omega_{m} \rho_{c}}\right)^{2} \int d M \frac{d n(M, z)}{d M} \tilde{u}(k \mid M) b_{1}(M, z)\right.} \\
& \left.\times\left(1+b_{\mathrm{sh}}(M)\right) \int d V \rho_{\text {host }}^{2}(r \mid M)\right]^{2} P_{\text {lin }}(k, z),
\end{aligned}
$$

where $P_{\text {lin }}(k, z)$ is the linear power spectrum of the matter density field $\delta$, and $b_{1}(M, z)$ is the linear halo bias [32]. The power spectrum of $\delta^{2}, P_{\delta^{2}}$, depends on profiles of density squared in a halo of mass $M, u(r \mid M)$, where $u(r \mid M)$ is normalized such that its volume integration becomes unity; $\tilde{u}(k \mid M)$ is the Fourier transform of $u(r \mid M)$.

Fourier transform of the density-squared profile, $\tilde{u}(k \mid M)$, is the sum of the density-squared profiles of the host halo and subhalos weighed by a fractional luminosity of each component:

$$
\tilde{u}(k \mid M)=\frac{\tilde{u}_{\text {host }}(k \mid M)+b_{\mathrm{sh}}(M) \tilde{u}_{\mathrm{sh}}(k \mid M)}{1+b_{\mathrm{sh}}(M)} .
$$




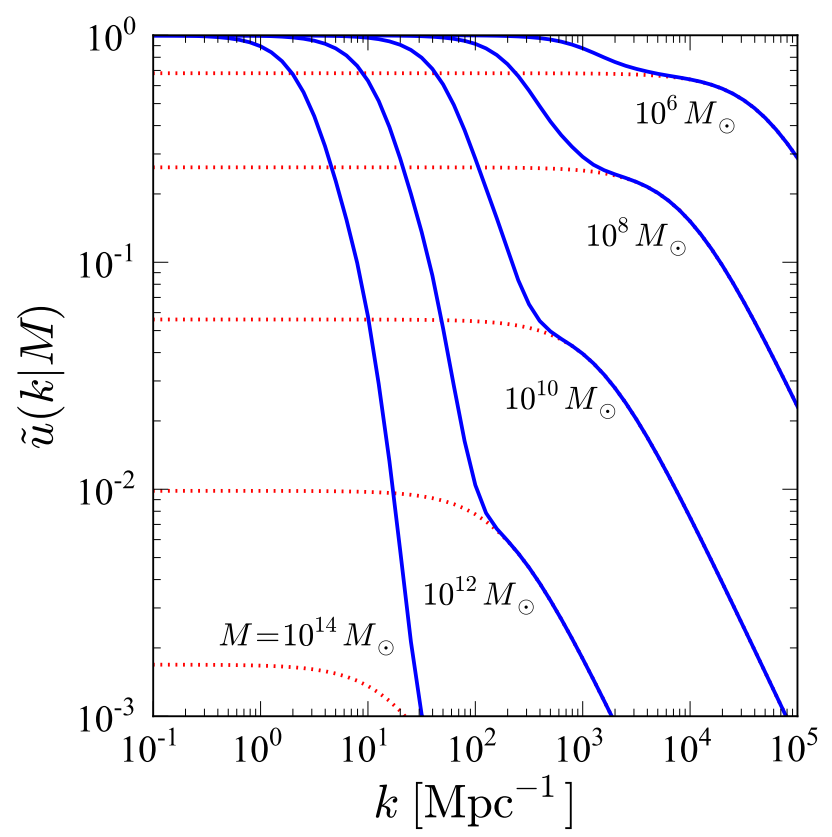

FIG. 5. Fourier transform of the density-squared profile, $\tilde{u}(k \mid M)$, for various host-halo masses. The solid lines show the total (host halo and subhalos) profile, whereas the dotted lines show the host-halo profiles. Note that the total $\tilde{u}(k \mid M)$ is normalized to unity at $k \rightarrow 0$, and thus the dotted lines do not approach unity at $k \rightarrow 0$ but approaches $1 /\left(1+b_{\mathrm{sh}}\right)$ [see Eq. [12].

Here, we ignore a contribution from the cross term, $2 \rho_{\text {host }}(r) \rho_{\text {sh }}(r)$, which is important only when $\rho_{\text {host }}(r) \sim$ $\rho_{\mathrm{sh}}(r)$ at the same radius, $r$. Given that spatial distributions of the host halo and subhalo contributions are quite different (the host halo being important inside the scale radius and the subhalos being important outside), this approximation is very good. (See Appendix for the contribution of the cross term.)

Fourier transform of the host halo profile, $u_{\text {host }}(r) \propto$ $\rho_{\text {host }}^{2}(r)$, has an analytic form [9], but here we use an even simpler fitting formula,

$$
\tilde{u}_{\text {host }}(k \mid M)=\frac{1}{\left[1+a\left(k r_{s}\right)^{2 / b}\right]^{b}},
$$

with $a=0.13$ and $b=0.7$, which is largely independent of $c_{\text {vir }}$ 20]. On the other hand, we obtain the density-squared profile of subhalos, $u_{\mathrm{sh}}(r \mid M)$, by deprojecting the surface brightness profiles of numerical simulations [29, 45], assuming spherical symmetry as follows:

$u_{\mathrm{sh}}(r) \propto\left\{\begin{array}{cl}{\left[\left(\frac{r}{r_{200}}\right)^{2}+\frac{1}{16}\right]^{-3 / 2},} & \text { for } r \leq r_{200}, \\ \left(\frac{16}{17}\right)^{3 / 2}\left(\frac{r}{r_{200}}\right)^{-1} e^{-\eta\left(r / r_{200}-1\right)}, & \text { for } r>r_{200},\end{array}\right.$

with $\eta=2.78$. Note that the distribution of subhalos is typically more extended than the density profile of the

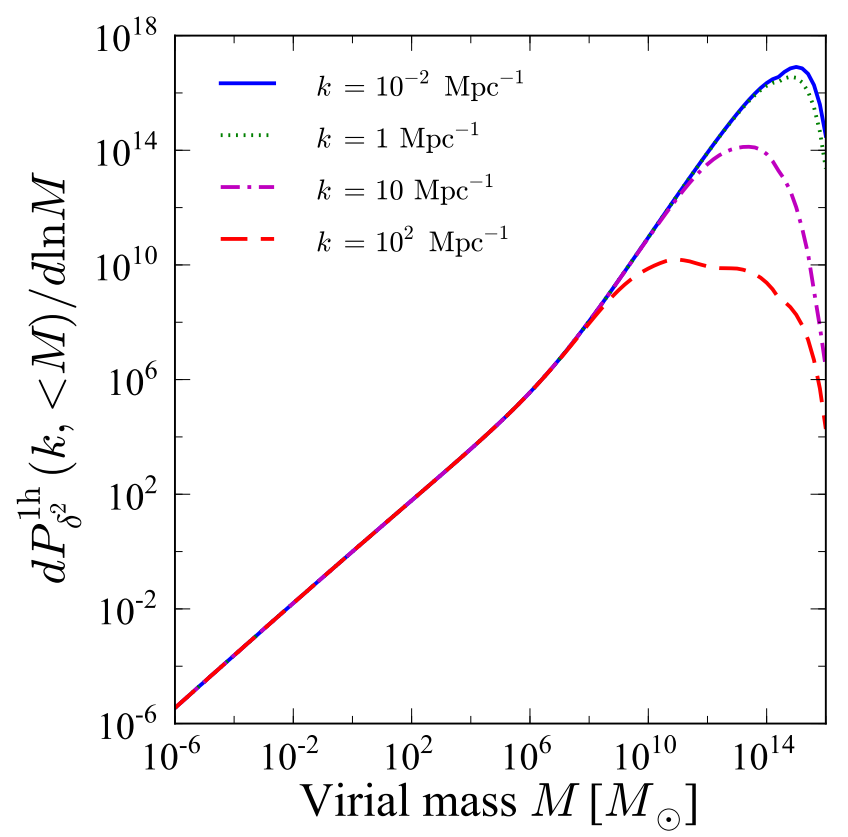

FIG. 6. Relative contributions to the one-halo power spectrum $P_{\delta^{2}}^{1 h}$ at $z=0$ as a function of masses and wave numbers. The lines are normalized at $M=1 M_{\odot}$.

host halo. Its Fourier transform is

$$
\begin{aligned}
\tilde{u}_{\mathrm{sh}}(k \mid M)= & A\left[\int_{0}^{1} d x \frac{x^{2}}{\left(x^{2}+1 / 16\right)^{3 / 2}} \frac{\sin (\kappa x)}{\kappa x}\right. \\
& \left.+\frac{64}{17^{3 / 2}} \frac{\kappa \cos \kappa+\eta \sin \kappa}{\kappa\left(\kappa^{2}+\eta^{2}\right)}\right]
\end{aligned}
$$

where $\kappa \equiv k r_{200}$ and $A \approx 0.64$ is the normalization constant such that $\tilde{u}_{\mathrm{sh}}(0)=1$.

Figure 5 shows $\tilde{u}(k \mid M)$ for various host-halo masses, $M$. When $\tilde{u}(k)$ is close to unity (for small $k$ ), the halo can be regarded as a point source. On the other hand, when $\tilde{u}(k)$ deviates significantly from unity at a given wave number $k$, the source extension cannot be ignored at that wave number, and the power spectrum (especially the one-halo term) is suppressed. Figure 5 shows that $\tilde{u}(k)$ is larger for smaller host halos, which are less extended. It also shows that the contributions from subhalos are more important for larger host halos. As the distribution of subhalos is more extended than the density profile of the host halo, the subhalo contribution dominates at small $k$ and the host-halo dominates at large $k$. This makes a hump at scales corresponding to the scale radius, $r_{s}$. In other words, annihilation from the smooth host-halo component dominates inside the scale radius, where subhalos are tidally disrupted.

Figure 6 shows the integrand of the one-halo power spectrum of $\delta^{2}, d P_{\delta^{2}}^{1 h}(k,<M) / d \ln M$ [Eq. [10]], at $z=0$ for various wave numbers, $k$. (Note that the lines are normalized at $M=1 M_{\odot}$, and thus it shows relative contributions rather than absolute.) The bulk of the con- 


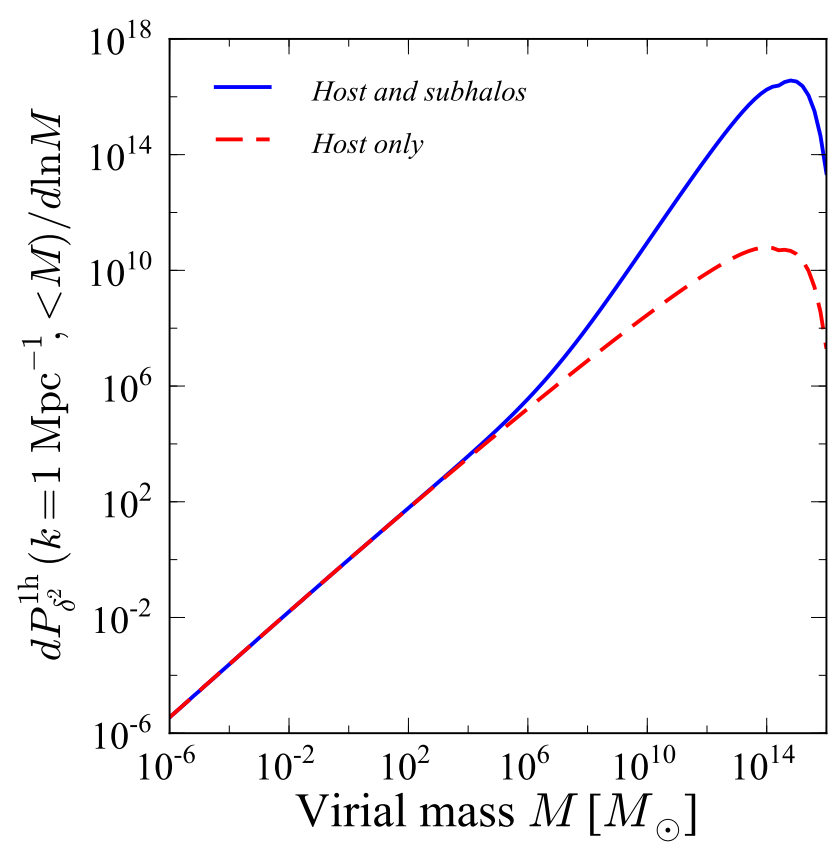

FIG. 7. The same as Fig. 6 for $k=1 \mathrm{Mpc}^{-1}$, but in comparison with the host-halo contribution.

tributions come from large-mass halos, and halos smaller than a typical dwarf size $\left(M<10^{6} M_{\odot}\right)$ do not make any sizable contributions to any relevant ranges of $k$. This is particularly true for scales larger than a typical cluster size (i.e., $k \lesssim 1 \mathrm{Mpc}^{-1}$ ). For smaller scales, on the other hand, a relative importance of large-mass halos is smaller, as large-mass halos are more extended and thus the power from them is suppressed (as also shown in Fig. 5).

Figure 7 is the same as Fig. 6 for $k=1 \mathrm{Mpc}^{-1}$, but the host-halo contribution is also shown. We find that the impact of subhalos on the power spectrum is much greater than that on the mean intensity (as shown in Fig. 2), and it can boost the power spectrum by almost four orders of magnitude at this particular wave number.

After projected on the sky, the three-dimensional wave number, $k$, and the angular multipole, $\ell$, are related by $k=\ell / \chi(z)$ for a given redshift, $z$ [see Eq. (7)]. Note, however, that this simple relation is valid only for small angular scales, $\ell \gg 1$ (e.g., [9, 10]), on which we mainly focus in this paper.

Figures 8 and 9 show the contributions to the angular power spectra at $\ell=10,100$, and 1000 from one-halo and two-halo terms, respectively, as a function of redshifts. To calculate a contribution to $C_{\ell}$ from a given $z$, we multiply $P_{\delta^{2}}$ by some combination of functions of redshift [see the integrand of Eq. (7) and also the redshift dependence in Eq. (2)]. We find that lower multipoles are dominated by nearby sources: one-halo terms at $\ell=10$, 100 , and 1000 are dominated by sources at $z \sim 0.002$, 0.02 , and 0.2 , respectively, whereas the dominant contributions to the two-halo term come from somewhat higher

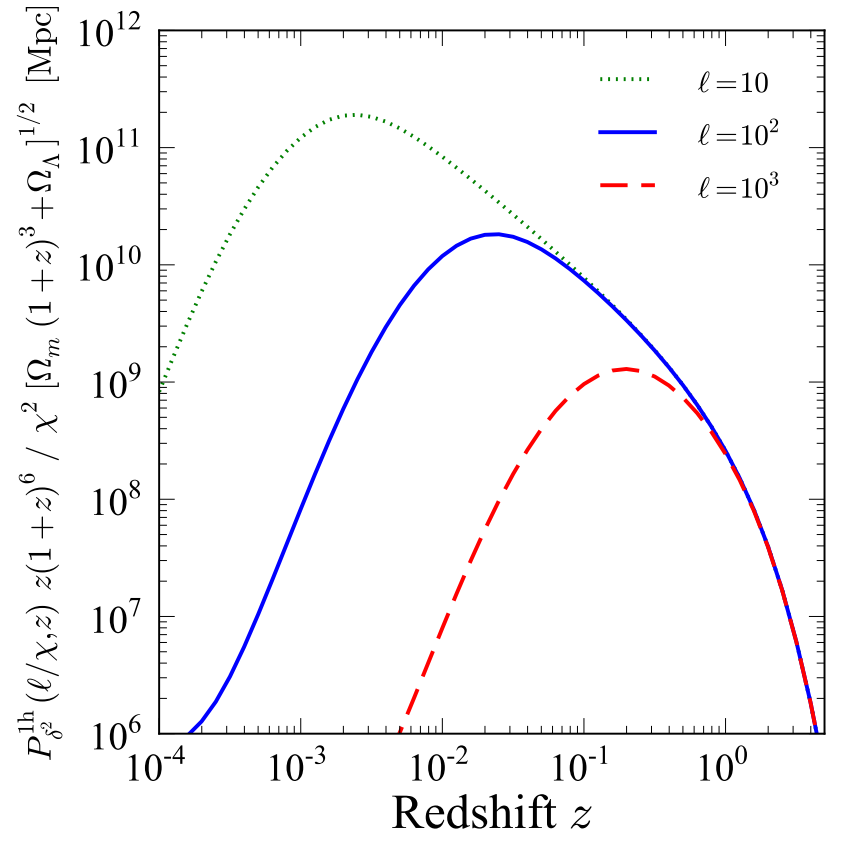

FIG. 8. One-halo contributions to the angular power spectrum, $C_{\ell}^{1 \mathrm{~h}}$, at $\ell=10,100$, and 1000 as a function of redshifts.

redshifts.

One should not, however, include contributions from arbitrarily small redshifts in the integral of Eq. (7), as cosmic variance in such small redshifts is so large that taking the ensemble average (as we do here) no longer makes sense. In addition, when a source is sufficiently close, it should give enough gamma-ray fluxes to be identified as an individual source which we can remove from the map. In the following discussion, we use three different minimum redshifts in the integration of Eq. (7): $z_{\min }=0.001,0.003$, and 0.01 . The r.m.s. overdensity within radii corresponding to these three redshifts are $1.4,0.74$, and 0.28 , respectively. Since none of these are much larger than one, we can argue that our results by setting these lower cutoffs are not subject to strong cosmic variance. We also note that, as we show below, this choice does not make any significant difference for the multipoles we consider $(\ell>150)$ in this paper.

In Fig. 10, we show both the one-halo and two-halo terms of the angular power spectrum, $C_{\ell}$, integrated over 5-10 GeV energy range, for three different values of $z_{\min }=0.001,0.003$, and 0.01 . The particle physics parameters are $\langle\sigma v\rangle=3 \times 10^{-26} \mathrm{~cm}^{3} \mathrm{~s}^{-1}$ and $m_{\mathrm{dm}}=100 \mathrm{GeV}$, and we assume the $b \bar{b}$ annihilation channel. Note that $C_{\ell}$ scales as $\langle\sigma v\rangle^{2}$. Taking smaller $z_{\text {min }}$ increases the power at large angular scales, in particular for the one-halo term, because of the contributions from closer, more extended halos. For the rest of this paper, we shall use $z_{\text {min }}=0.003$ for definiteness.

The thick solid line in Fig. 10 shows the upper limits on the angular power spectrum [27] (with the blazar con- 


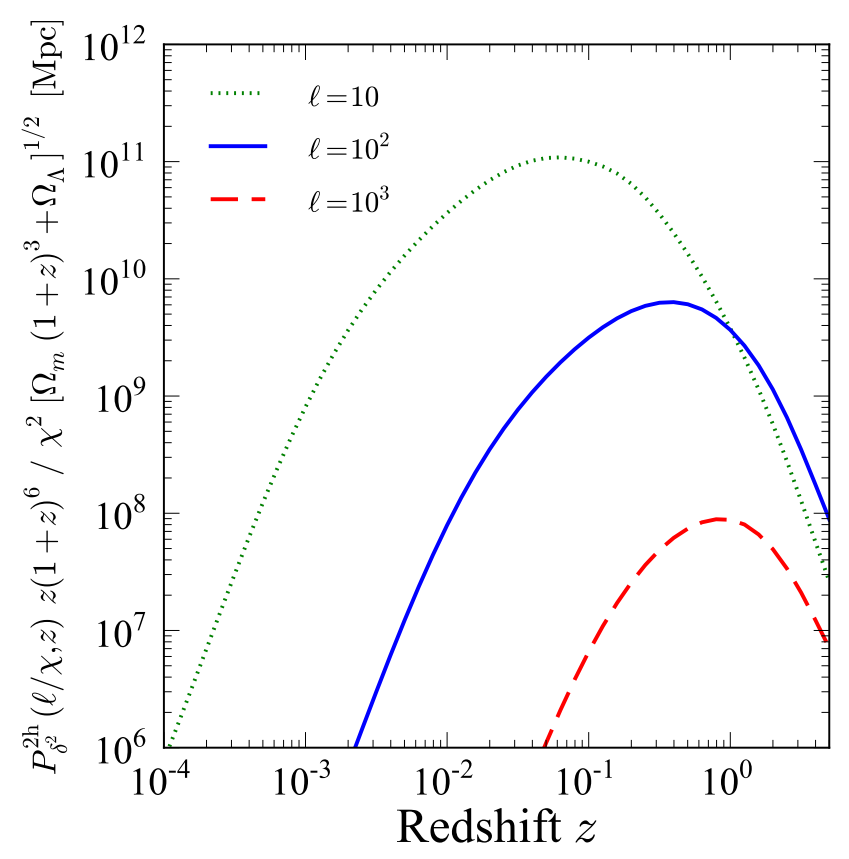

FIG. 9. The same as Fig. 8 but for the two-halo contributions to the angular power spectrum, $C_{\ell}^{2 \mathrm{~h}}$.

tribution subtracted [28]) between $\ell=155$ and 504 for 5 $10.4 \mathrm{GeV}, C_{155 \leq \ell \leq 504} \leq 8 \times 10^{-20}\left(\mathrm{~cm}^{-2} \mathrm{~s}^{-1} \mathrm{sr}^{-1}\right)^{2} \mathrm{sr}$. The current upper limits in $5-10.4 \mathrm{GeV}$, whose energy region is most sensitive to dark matter particles with $m_{\mathrm{dm}}=100 \mathrm{GeV}$ annihilating into $b \bar{b}$ (see Fig. 4), are three orders of magnitude larger than the prediction with the canonical particle physics parameters. Recalling $C_{\ell} \propto\langle\sigma v\rangle^{2}$, we find an upper limit on the cross section of $\langle\sigma v\rangle \lesssim 8 \times 10^{-25} \mathrm{~cm}^{3} \mathrm{~s}^{-1}$ for the $100-\mathrm{GeV}$ dark matter annihilating into $b \bar{b}$.

The blazar-subtracted upper limits on the angular power spectrum are available in several energy bands, 1.04-1.99 GeV, 1.99-5 GeV, 5-10.4 GeV, and 10.4$50 \mathrm{GeV}$ 28. In Fig. 11, we show the combined upper limits on the annihilation cross section for the $b \bar{b}$ channel, $\langle\sigma v\rangle_{b \bar{b}}$, as a function of the dark matter masses, $m_{\mathrm{dm}}$, using all the available data on the power spectrum. (Note that the model is still based only on the extragalactic contribution.) We calculate the upper limit on the cross section such that the predicted $C_{\ell}$ at $\ell=155$ (the lowest multipole at which the measurement is reported) is equal to the $2 \sigma$ upper limit reported by Ref. [28]. The limits from each energy range are shown separately as the dotted lines. The combined limits shown as the solid line are simply the best of the four limits at a given dark matter mass. A more optimal analysis would improve these limits.

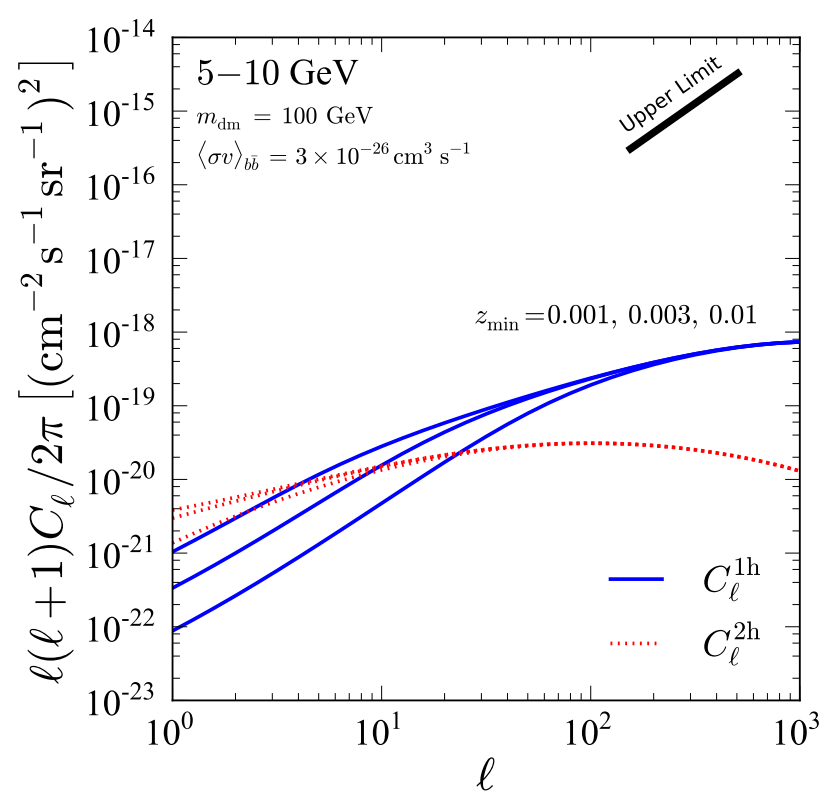

FIG. 10. Predicted angular power spectrum of DGRB from dark matter annihilation in extragalactic halos, integrated over the energy range between $5 \mathrm{GeV}$ and $10 \mathrm{GeV}$. (This energy range is most sensitive to dark matter particles with $m_{\mathrm{dm}}=100 \mathrm{GeV}$ annihilating into $b \bar{b}$; see Fig. 4.) The onehalo (solid) and two-halo (dotted) terms are shown separately, for three different minimum redshifts $z_{\min }=0.003,0.001$, and 0.01 (from top to bottom lines). The particle physics parameters are $\langle\sigma v\rangle=3 \times 10^{-26} \mathrm{~cm}^{3} \mathrm{~s}^{-1}$ and $m_{\mathrm{dm}}=100 \mathrm{GeV}$, and the $b \bar{b}$ annihilation channel is assumed. For comparison, the thick solid line shows the upper limit on the angular power spectrum in $155 \leq \ell \leq 504$ from the 22-month data of FermiLAT 27] with the blazar contribution subtracted 28].

\section{CROSS CORRELATION BETWEEN DARK MATTER AND BLAZARS}

As astrophysical gamma-ray sources, such as blazars associated with supermassive black holes at the center of galaxies, reside in dark matter halos, there is a spatial correlation between gamma rays from dark matter annihilation and blazars. The angular power spectrum of dark matter annihilation including the cross correlation, which is equal to the total power spectrum minus the blazar power spectrum, is given by

$$
C_{\ell}^{\mathrm{dm}} \equiv C_{\ell}-C_{\ell}^{\text {blazar }}=C_{\ell}^{1 \mathrm{~h}}+C_{\ell}^{2 \mathrm{~h}}+2 C_{\ell}^{\times},
$$




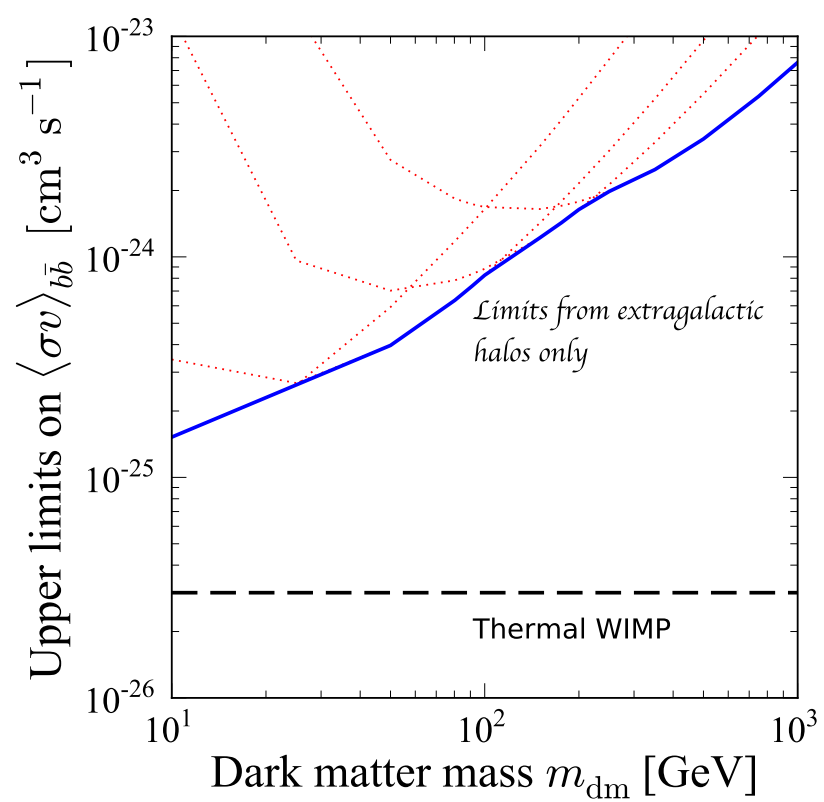

FIG. 11. Upper limits on the annihilation cross section into the $b \bar{b}$ final state as a function of the dark matter masses, $m_{\mathrm{dm}}$. The limits are obtained from the measurement of the angular power spectrum at $\ell=155$ [27] with the blazar contribution subtracted 28, and the theoretical model of dark matter annihilation from extragalactic halos. The dotted lines show the limits from different energy ranges: $1.04-1.99 \mathrm{GeV}$, 1.99-5 GeV, 5-10.4 GeV, and $10.4-50 \mathrm{GeV}$ from bottom to top at low-mass region. The solid line shows the combined limits, and the horizontal dashed line shows the canonical cross section for the thermal-freezeout scenario.

where $C_{\ell}^{\times}$is the cross-power spectrum computed from ${ }^{3}$

$$
\begin{aligned}
C_{\ell}^{\times}(E)= & \int \frac{d \chi}{\chi^{2}} W_{B}([1+z] E, z) W_{\mathrm{dm}}([1+z] E, z) \\
& \times P_{\times}\left(k=\frac{\ell}{\chi}, z\right),
\end{aligned}
$$

where the subscripts " $B$ " and "dm" denote blazars and dark matter, respectively. Here, $W_{\mathrm{dm}}$ is the same as Eq. (2), while $W_{B}$ is the window function for the mean intensity of blazars [similarly defined as Eq. (1) but replacing $\left\langle\delta^{2}\right\rangle$ with 1]:

$$
W_{B}([1+z] E, z)=\chi^{2} \int_{0}^{\mathcal{L}\left(\mathcal{F}_{E, \lim }, z\right)} d \mathcal{L} \Phi_{E}(\mathcal{L}, z) \mathcal{F}_{E}(\mathcal{L}, z),
$$

where $\mathcal{L}$ is the differential luminosity (i.e., luminosity per unit energy) at a given energy $E, \mathcal{F}_{E}$ is the differential

\footnotetext{
${ }^{3}$ We include blazars in the cross-correlation, ignoring other sources of gamma rays. This may be justified, as blazars are so far known to be the most dominant extragalactic gamma-ray sources in the $\mathrm{GeV}$ energy regime. Other promising sources include star-forming galaxies 21 46, 48.
}

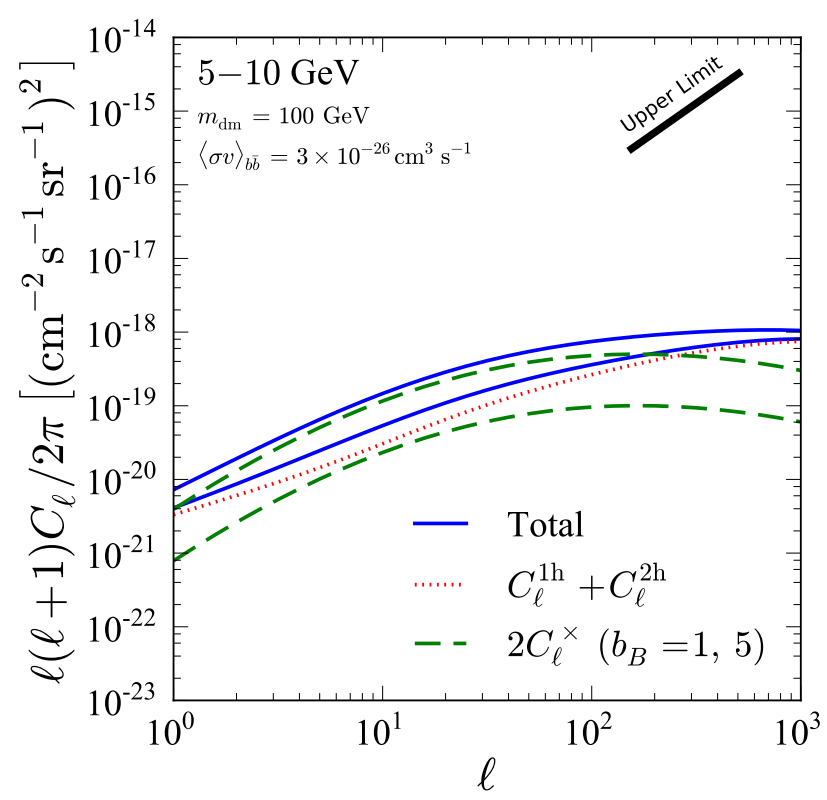

FIG. 12. Predicted angular power spectra of DGRB in 5$10 \mathrm{GeV}$ from dark matter annihilation only (dotted), dark matter-blazar cross correlation (dashed), and the sum of the two (solid). The particle-physics parameters are the same as those in Fig. 10. For the blazar bias, we use $b_{B}=1$ (bottom dashed/solid) and 5 (top dashed/solid).

flux, and $\Phi_{E}(\mathcal{L}, z)$ is the gamma-ray luminosity function of blazars. The upper limit of the integral, $\mathcal{L}\left(\mathcal{F}_{E}, z\right)$, is the luminosity giving the flux corresponding to the point-source sensitivity of Fermi-LAT at a given redshift $z$, for which we adopt $10^{-8} \mathrm{~cm}^{-2} \mathrm{~s}^{-1}$ above $100 \mathrm{MeV}$. More formal definitions of these quantities can be found in Ref. [10.

The three-dimensional cross power spectrum is

$$
\begin{aligned}
P_{\times}(k, z)= & \left\langle b_{B}(z)\right\rangle\left[\left(\frac{1}{\Omega_{m} \rho_{c}}\right)^{2} \int d M \frac{d n}{d M}(M, z) \tilde{u}(k \mid M)\right. \\
& \left.\times b_{1}(M, z)\left(1+b_{\mathrm{sh}}(M)\right) \int d V \rho_{\text {host }}^{2}(r \mid M)\right] \\
& \times P_{\text {lin }}(k, z) \\
= & \left\langle b_{B}(z)\right\rangle \sqrt{P_{\delta^{2}}^{2 \mathrm{~h}}(k, z) P_{\text {lin }}(k, z)},
\end{aligned}
$$

where $\left\langle b_{B}(z)\right\rangle$ is the blazar bias averaged over the luminosity function and the flux as follows:

$$
\left\langle b_{B}(z)\right\rangle=\frac{\int d \mathcal{L} \Phi_{E}(\mathcal{L}, z) \mathcal{F}_{E}(\mathcal{L}, z) b_{B}(\mathcal{L}, z)}{\int d \mathcal{L} \Phi_{E}(\mathcal{L}, z) \mathcal{F}_{E}(\mathcal{L}, z)},
$$

with the luminosity-dependent bias, $b_{B}(\mathcal{L}, z)$, and the same upper and lower limits of integration as those in Eq. (18).

Note that this power spectrum [Eq. (19)] includes the two-halo term only. While the one-halo term, where dark matter annihilation happens in the same halo that hosts 
a blazar, also exists, the previous study [10] shows that the one-halo term of the cross correlation is much smaller than the two-halo term; thus, we shall ignore the one-halo term of the cross-power spectrum.

For the luminosity function, $\Phi_{E}(\mathcal{L}, z)$, we adopt the luminosity-dependent density-evolution (LDDE) model [11, 49] with the gamma-ray spectra assumed to be a power law with an index of 2.4 , which is in agreement with the spectrum of resolved blazars as well as that of the DGRB ${ }^{4}$ Compared with the earlier study [11] where the luminosity function was based on pre-Fermi data, we here adopt different values for parameters of the luminosity function $\left(\kappa=10^{-4}, q=3.5\right.$, and $\left.\gamma_{1}=1.05\right)$ such that the model reproduces the flux distribution of blazars resolved by Fermi 44 .

In Fig. 4, we show the blazar contribution to the mean intensity. It is difficult to explain the DGRB intensity measured by Fermi with blazars alone, in agreement with the previous study [44, 52.

Figure 12 shows the angular power spectra from the cross correlation with $\left\langle b_{B}\right\rangle=1$ and 5 , for the energy range between $5 \mathrm{GeV}$ and $10 \mathrm{GeV}$. The particle-physics parameters are the same as those in Fig. 10. We find that, if the annihilation cross section is around the canonical value required to produce dark matter particles at the right abundance by the thermal-freezeout mechanism, then the cross-correlation term cannot be ignored. This is particularly important when the bias of blazars is as high as 5 .

As the dark matter term $\left(C_{\ell}^{1 \mathrm{~h}}+C_{\ell}^{2 \mathrm{~h}} \propto\langle\sigma v\rangle^{2}\right)$ and the cross-correlation term $\left(C_{\ell}^{\times} \propto\langle\sigma v\rangle\right)$ scale with $\langle\sigma v\rangle$ differently, one may ask, "At which value of the annihilation cross section does the cross-correlation term become important?" Figure 13 shows $C_{\ell=155}$ divided by the upper limit, $C_{155 \leq \ell \leq 504}^{\mathrm{UL}}$, for $5-10 \mathrm{GeV}$. The dashed line is without the cross correlation, while the solid lines are with the cross correlation with bias of 1 and 5 . We find that the dark matter term dominates at $\langle\sigma v\rangle \gtrsim 3 \times 10^{-26} \mathrm{~cm}^{3} \mathrm{~s}^{-1}$, whereas the cross-correlation term dominates at lower cross sections.

\section{GALACTIC CONTRIBUTION}

Annihilation signals from subhalos in the Galactic halo containing the Milky Way are typically comparable to, or even greater than, the extragalactic contribution [7, 8, 15, 18, 20; thus, we must also take the Galactic subhalo contribution into account. We shall follow an analytic treatment presented in Ref. [20].

On the other hand, we do not include the contribution from a smooth density profile of the host halo of the Milky Way in our calculation. In the anisotropy analysis

\footnotetext{
${ }^{4}$ More elaborated spectra in combination with the luminosity function and the DGRB intensity are studied in Refs [50, 51].
}

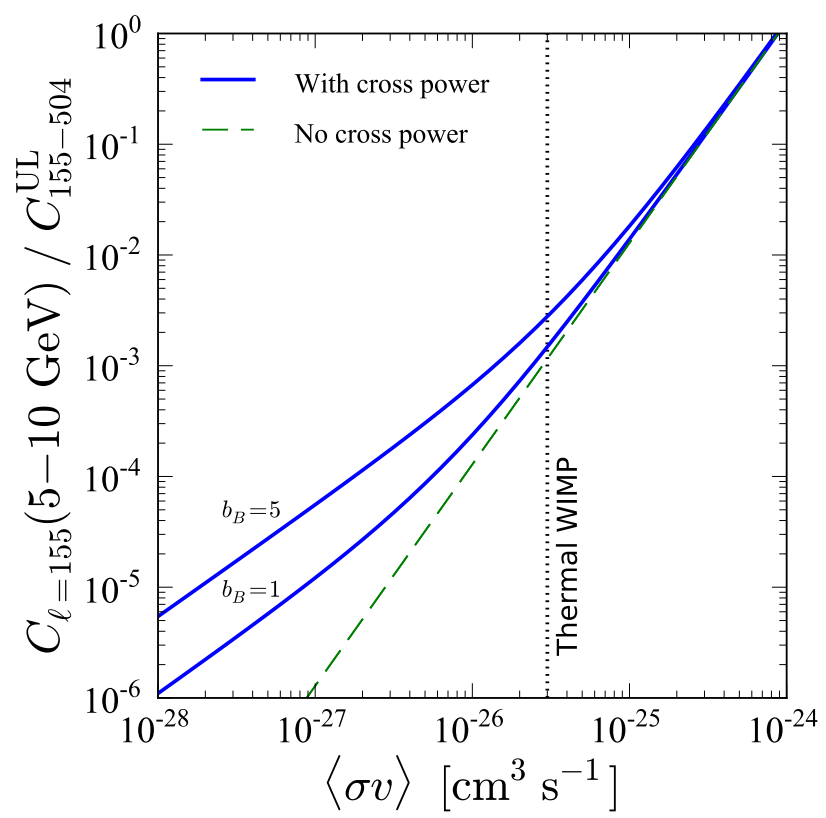

FIG. 13. Predicted angular power spectrum at $\ell=155$ in $5-$ $10 \mathrm{GeV}$ divided by the current upper limit, $C_{155 \leq \ell \leq 504}^{\mathrm{UL}}$, as a function of the annihilation cross section, $\langle\sigma v\rangle$. The solid lines include the dark-matter-blazar cross-correlation with $\left\langle b_{B}\right\rangle=$ 1 and 5, while the dashed line does not. The vertical dotted line shows the canonical cross section for thermal WIMPs.

of the Fermi-LAT data [27], the low Galactic latitude region of $|b| \leq 30 \mathrm{deg}$ is masked. Using a density profile of the smooth Galactic component of Ref. [20], we find that this mask brings the smooth contribution to the mean intensity down to about $10 \%$ of the subhalo contribution, as shown in Fig. 14. As the smooth component does not have much power on small angular scales, it can be safely ignored for $\ell \gtrsim 100$ (but it can be comparable to the subhalo and extragalactic contributions on large angular scales, $\ell \lesssim 10)$.

The angle-averaged mean intensity from dark matter annihilation in Galactic subhalos can be computed from

$$
I_{\mathrm{sh}}=\int d L \int_{s_{*}(L)}^{r_{\mathrm{vir}, \mathrm{MW}}} d s \frac{d \overline{n_{\mathrm{sh}}}(L, s)}{d L} L,
$$

where $L$ is the gamma-ray luminosity of a subhalo, $s$ is the line-of-sight coordinates, and $d \overline{n_{\mathrm{sh}}} / d L$ is the angleaveraged luminosity function of subhalos. The lower limit of the line-of-sight integral, $s_{*}(L)$, corresponds to the flux sensitivity of Fermi-LAT, i.e., $L=4 \pi s_{*}^{2} F_{\text {sens }}$.

The subhalo luminosity, $L$, is related to the subhalo mass, $M$, via

$$
L=B_{\mathrm{sh}} \frac{\langle\sigma v\rangle N_{\gamma, \text { ann }}}{2 m_{\mathrm{dm}}^{2}} \int d V_{\mathrm{sh}} \rho_{\mathrm{sh}}^{2}\left(r_{\mathrm{sh}} \mid M\right),
$$

where $r_{\text {vir,MW }}$ is the virial radius of the Galactic halo, the subscripts "sh" denote subhalos, and $B_{\text {sh }}$ is a boost 


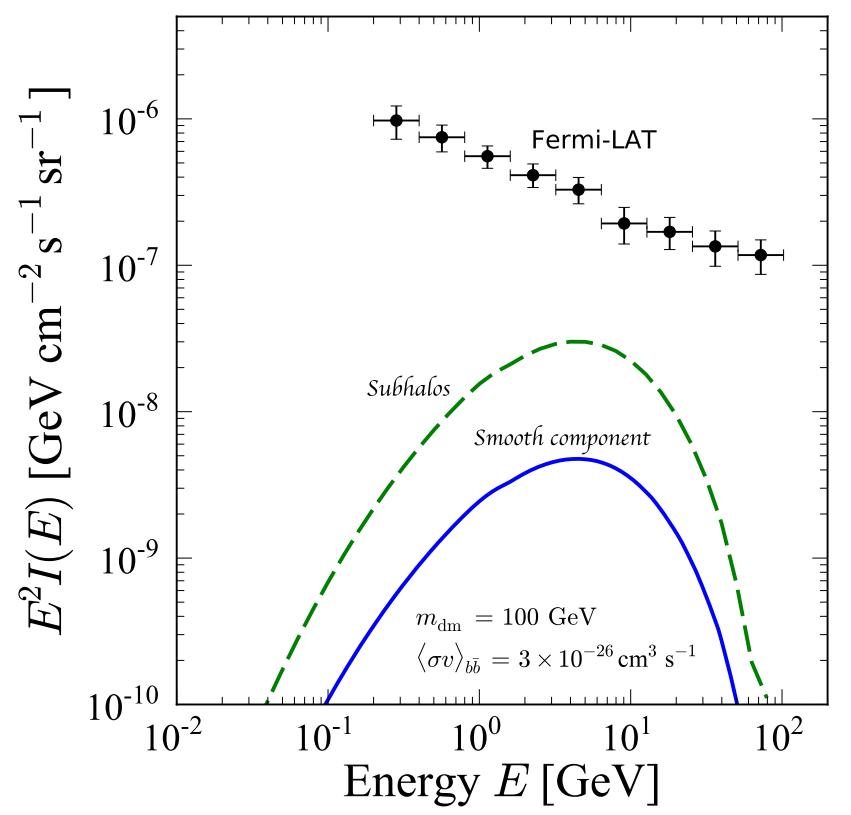

FIG. 14. The same as Fig. 4, but for the Galactic smooth component in the high Galactic latitude region outside of $|b|>$ $30 \mathrm{deg}$ (solid). The dashed line is the same as that in Fig. 4

factor due to the presence of substructure in subhalos (sub-subhalos).

We assume that the density profile of subhalos, $\rho_{\text {sh }}$, is well described by the NFW function [Eq. (4)]. Then, the volume integral of the density-squared has the analytic form given by Eq. (6) with the concentration parameter, $c_{\text {vir }}$, replaced with $c_{\text {cut }}$ that corresponds to the cutoff radius of subhalos, i.e., $c_{\text {cut }} \equiv r_{\text {cut }} / r_{s}$. With this massluminosity relation [Eq. (22)] and the subhalo mass function $d n_{\mathrm{sh}} / d M$, one can compute the luminosity function. Most model inputs such as the subhalo mass function, spatial distribution, and mass-concentration relation are adopted from recent numerical simulations of the Galactic halo, Aquarius [53]. More details on how to apply these models to gamma-ray computations are described in Ref. [20.

The intensity angular power spectrum is

$$
C_{\ell}^{\mathrm{sh}}=\frac{1}{16 \pi^{2}} \int d L \int \frac{d s}{s^{2}} L^{2} \frac{d \overline{n_{\mathrm{sh}}}(L, s)}{d L}\left|\tilde{u}_{\mathrm{sh}}\left(\frac{\ell}{s}, M\right)\right|^{2},
$$

where $\tilde{u}_{\mathrm{sh}}(k, M)$ is the Fourier transform of the densitysquared profile of the subhalo distribution, which is given by Eq. 13 if the density distribution of subhalos follows an NFW profile. Note that Eq. 23) only includes "onesubhalo" term, where one correlates two points in one identical subhalo. There is, however, the two-subhalo term that correlates two points in two distinct subhalos, but this term is much smaller than the one-subhalo term at small angular scales 20.

Figure 15 shows the predicted angular power spectra

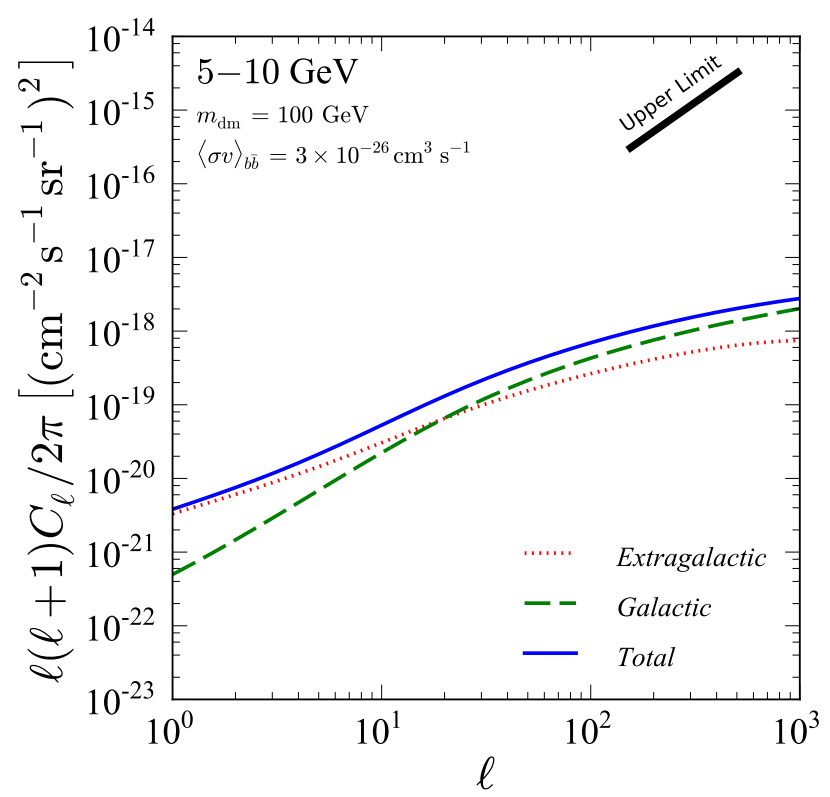

FIG. 15. Predicted angular power spectra of DGRB in 5$10 \mathrm{GeV}$ from dark matter annihilation in extragalactic halos (dotted), Galactic subhalos (dashed), and the sum of the two (solid).

from Galactic subhalos and extragalactic halos (but not including the cross correlation). We have used the canonical model of the Galactic subhalos given in Ref. [53, which has the mass resolution of about $4 \times 10^{4} M_{\odot}$. We have extrapolated their result down to the Earth-mass scale (model A1 of Ref. 20]). The intensity power spectrum is about the same for both the extragalactic and Galactic components, with the latter slightly larger in the angular scales constrained by Fermi-LAT.

In Fig. 16, we show the limits on $\langle\sigma v\rangle$ from the FermiLAT data, taking into account both the extragalactic and Galactic terms. As expected, the limits from either alone are similar, and the combined limits improve by a factor of two. In particular, for low-mass dark matter particles, the combined limits are only a factor of three larger than the canonical cross section. The limits are weaker for larger masses.

While our limits are not yet as stringent as those obtained from analyses of dwarf galaxies [54, 55] or galaxy clusters [45, 56, where the canonical cross section is already excluded for low-mass $(\sim 10 \mathrm{GeV})$ dark matter particles, they are not so far away (i.e., only a factor of three to four worse). Also, our limits are derived in a completely different way: they are based on the diffuse emission rather than on individual objects, and they are based on anisotropy rather than on the mean intensity. It is certainly encouraging that the first limits using the DGRB anisotropy are already not so far away from the best limits. 


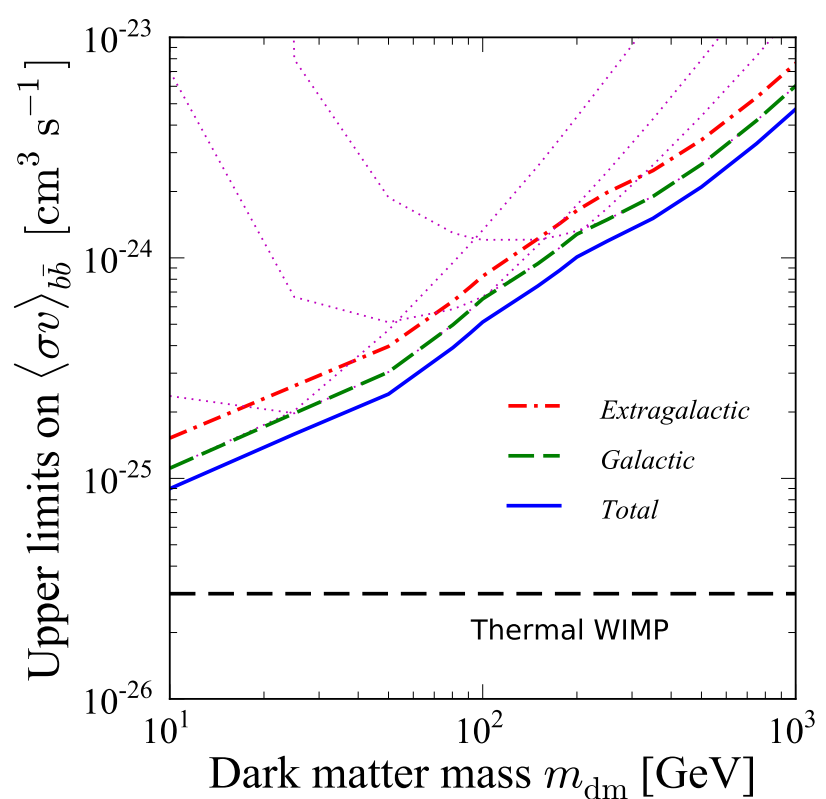

FIG. 16. The same as Fig. 11, but for the limits obtained from the Galactic subhalos (dashed); extragalactic halos (dotdashed); and the sum of the two (solid). The dot-dashed line is the same as the solid line in Fig. 11. The dotted lines show the Galactic-subhalo limits from each of four energy bins.

\section{CONCLUSIONS}

In this paper, we have used the angular power spectrum of DGRB recently detected in the 22-month data of Fermi-LAT [27] to place limits on the annihilation cross section of dark matter particles as a function of dark matter masses. As dark matter annihilation occurs in all cosmological halos and subhalos, our model includes all the contributing terms in the extragalactic halos, the Galactic subhalos, and the cross correlation between dark matter annihilation and blazars. The smooth Galactic component is predicted to be sub-dominant in the high Galactic region $(|b|>30 \mathrm{deg})$ and is ignored.

We have revised our earlier model of the extragalactic contribution by including the results from recent numerical simulations of the subhalo distribution [29]. Combined with the model of the Galactic subhalos of Ref. [20], we find that the Galactic and extragalactic contributions are comparable to each other. The cross correlation with blazars is important for annihilation cross sections smaller than the canonical value $\left(\langle\sigma v\rangle \lesssim 3 \times 10^{-26} \mathrm{~cm}^{3} \mathrm{~s}^{-1}\right)$.

By comparing our model with the upper limit on the non-blazar contribution to the angular power spectrum of DGRB [28, we find upper limits on the annihilation cross section as a function of dark matter masses as shown in Fig. 16. The current limit from anisotropy excludes regions of $\langle\sigma v\rangle \gtrsim 10^{-25} \mathrm{~cm}^{3} \mathrm{~s}^{-1}$ at the dark matter mass of $10 \mathrm{GeV}$, which is only a factor of three larger than the

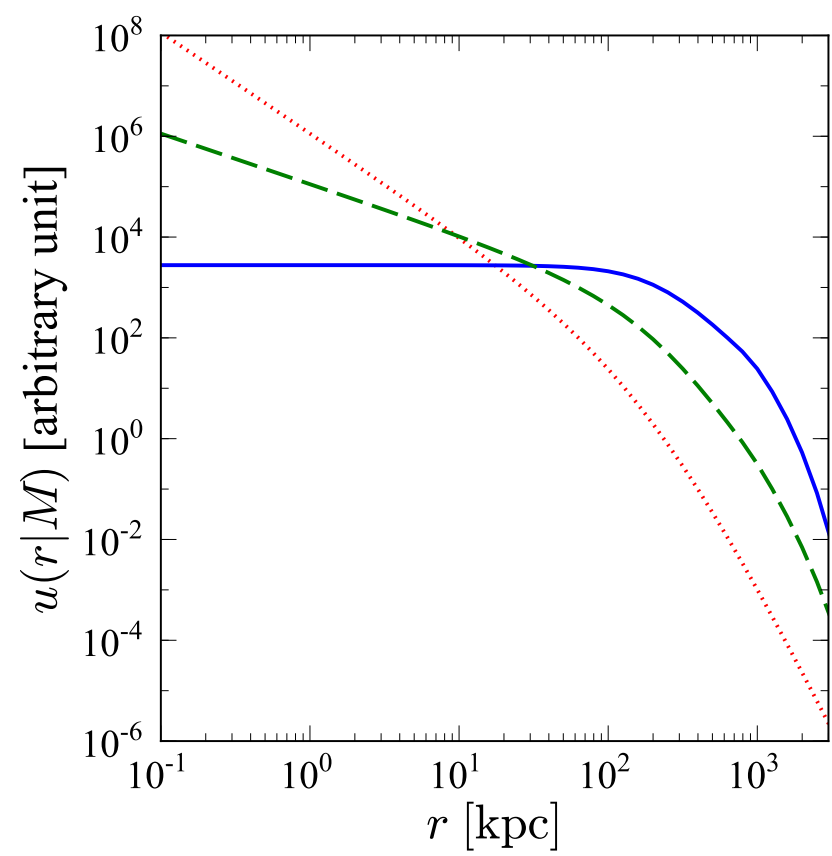

FIG. 17. Density-squared profiles of a host halo (dotted); subhalos (solid); and a host-subhalo cross-term (dashed). The mass of the host halo is $M=10^{14} M_{\odot}$ and the redshift is $z=0$. The virial radius is $1.2 \mathrm{Mpc}$ and the scale radius is $210 \mathrm{kpc}$.

canonical value. The limits are weaker for larger dark matter masses. The first limits from DGRB anisotropy that we find in this paper are already competitive with the best limits in the literature.

Our limits will improve as Fermi collects more data. At the same time, an improvement in the analysis can significantly improve our limits. Currently, the angular power spectrum on large angular scales, $\ell<155$, is not used because of a potential contamination by the Galactic foreground emission (such as pion decay). As the angular power spectrum of DGRB from dark matter annihilation, $C_{\ell}$ (without multiplying by $\ell^{2}$ ), rises towards low multipoles, including the low-multipole data will significantly improve the limits. This line of investigation (i.e., a better characterization and removal of the Galactic foreground) should be pursued.

\section{ACKNOWLEDGMENTS}

The work of S.A. was supported by GRAPPA Institute at University of Amsterdam and by NWO through Vidi Grant. 


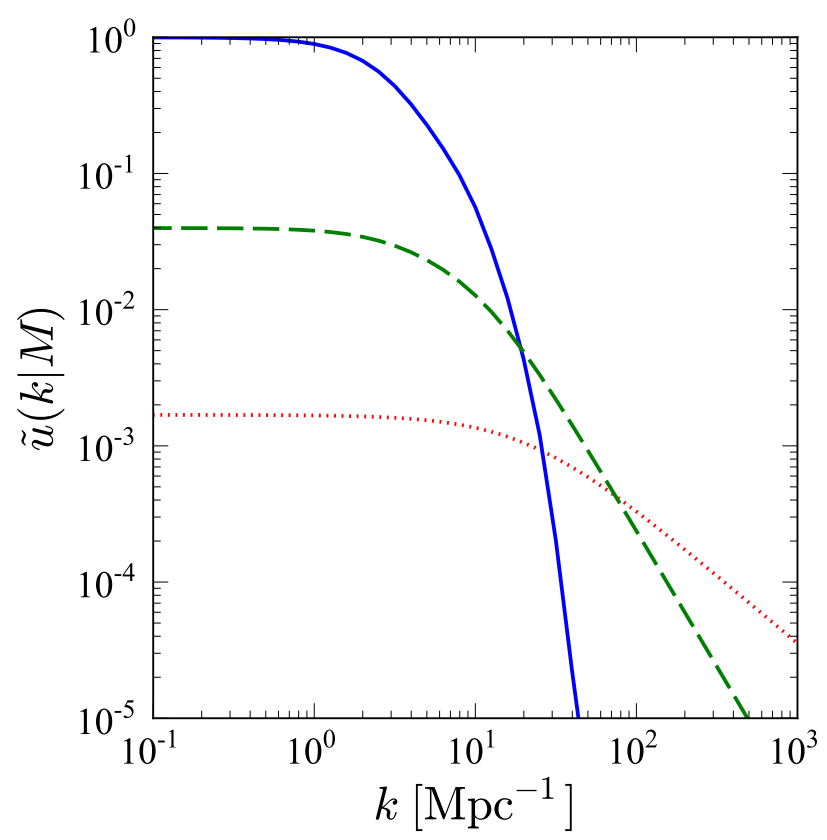

FIG. 18. Fourier transform of the lines in Fig. 17

\section{Appendix A: Effect of host-subhalo cross-term}

The dark matter annihilation signal is proportional to the density squared. When both host halo and subhalo contributions are present, one has the host-densitysquared term, $\rho_{\text {host }}^{2}$, the subhalo-density-squared term, $\rho_{\text {sh }}^{2}$, and the host-subhalo cross term, $2 \rho_{\text {host }} \rho_{\text {sh }}$. In our analysis, we have ignored the cross term [see Eq. (12)], as spatial distributions of the host halo and subhalo contributions are quite different (the host halo being important inside the scale radius and the subhalos being important outside). In this Appendix, we quantify the importance of the cross term.

Figure 17 shows the density-squared profiles of a host halo, subhalos, and the cross term. As expected, the cross term becomes comparable to the other terms only within a narrow window in radii. For the host halo mass of $M=10^{14} M_{\odot}$ and the redshift of $z=0$, the cross term becomes comparable to the other terms at $r \sim 20 \mathrm{kpc}$, which is $1 / 10$ of the scale radius, $r_{s}=210 \mathrm{kpc}$. Fig. 18 shows the Fourier transform.
[1] G. Jungman, M. Kamionkowski and K. Griest, Phys. Rept. 267 (1996) 195 hep-ph/9506380.

[2] L. Bergstrom, Rept. Prog. Phys. 63 (2000) 793 hep$\mathrm{ph} / 0002126$.

[3] G. Bertone, D. Hooper and J. Silk, Phys. Rept. 405 (2005) 279 hep-ph/0404175.

[4] P. Ullio, L. Bergstrom, J. Edsjo and C. G. Lacey, Phys. Rev. D 66 (2002) 123502 astro-ph/0207125.

[5] J. E. Taylor and J. Silk, Mon. Not. Roy. Astron. Soc. 339 (2003) 505 astro-ph/0207299.

[6] S. Ando, Phys. Rev. Lett. 94 (2005) 171303 astro$\mathrm{ph} / 0503006$.

[7] T. Oda, T. Totani and M. Nagashima, Astrophys. J. 633 (2005) L65 astro-ph/0504096.

[8] L. Pieri, G. Bertone and E. Branchini, Mon. Not. Roy. Astron. Soc. 384 (2008) 1627 arXiv:0706.2101 [astro$\mathrm{ph}]]$.

[9] S. Ando and E. Komatsu, Phys. Rev. D 73 (2006) 023521 astro-ph/0512217.

[10] S. Ando, E. Komatsu, T. Narumoto and T. Totani, Phys. Rev. D 75 (2007) 063519 astro-ph/0612467.

[11] S. Ando, E. Komatsu, T. Narumoto and T. Totani, Mon. Not. Roy. Astron. Soc. 376 (2007) 1635 astro$\mathrm{ph} / 0610155$.

[12] F. Miniati, S. M. Koushiappas and T. Di Matteo, Astrophys. J. 667 (2007) L1 astro-ph/0702083.

[13] A. Cuoco, S. Hannestad, T. Haugbolle, G. Miele, P. D. Serpico and H. Tu, JCAP 0704 (2007) 013 astro$\mathrm{ph} / 0612559$.

[14] A. Cuoco, J. Brandbyge, S. Hannestad, T. Haugboelle and G. Miele, Phys. Rev. D 77 (2008) 123518 arXiv:0710.4136 [astro-ph]].

[15] J. M. Siegal-Gaskins, JCAP $0810 \quad$ (2008) 040
arXiv:0807.1328 [astro-ph]].

[16] S. K. Lee, S. Ando and M. Kamionkowski, JCAP 0907 (2009) 007 arXiv:0810.1284 [astro-ph]].

[17] M. Taoso, S. Ando, G. Bertone and S. Profumo, Phys. Rev. D 79 (2009) 043521 arXiv:0811.4493 [astro-ph]].

[18] M. Fornasa, L. Pieri, G. Bertone and E. Branchini, Phys. Rev. D 80 (2009) 023518 arXiv:0901.2921 [astro-ph]].

[19] J. M. Siegal-Gaskins and V. Pavlidou, Phys. Rev. Lett. 102 (2009) 241301 arXiv:0901.3776 [astro-ph.HE]].

[20] S. Ando, Phys. Rev. D $\mathbf{8 0} \quad$ (2009) 023520 arXiv:0903.4685 [astro-ph.CO]].

[21] S. Ando and V. Pavlidou, Mon. Not. Roy. Astron. Soc. 400 (2009) 2122 arXiv:0908.3890 [astro-ph.HE]].

[22] J. Zavala, V. Springel and M. Boylan-Kolchin, Mon. Not. Roy. Astron. Soc. 405 (2010) 593 arXiv:0908.2428 [astro-ph.CO]].

[23] A. Ibarra, D. Tran and C. Weniger, Phys. Rev. D 81 (2010) 023529 arXiv:0909.3514 [hep-ph]].

[24] J. M. Siegal-Gaskins, R. Reesman, V. Pavlidou, S. Profumo and T. P. Walker, Mon. Not. Roy. Astron. Soc. 415 (2011) 1074S arXiv:1011.5501 [astro-ph.HE]].

[25] A. Cuoco, A. Sellerholm, J. Conrad and S. Hannestad, Mon. Not. Roy. Astron. Soc. 414 (2011) 2040 arXiv:1005.0843 [astro-ph.HE]].

[26] M. Fornasa et al., arXiv:1207.0502 [astro-ph.HE].

[27] M. Ackermann et al. [Fermi LAT Collaboration], Phys. Rev. D 85 (2012) 083007 arXiv:1202.2856 [astroph.HE]].

[28] A. Cuoco, E. Komatsu and J. M. Siegal-Gaskins, Phys. Rev. D 86 (2012) 063004 arXiv:1202.5309 [astroph.CO]].

[29] L. Gao, C. S. Frenk, A. Jenkins, V. Springel and S. D. M. White, Mon. Not. Roy. Astron. Soc. 419 (2012) 
1721 arXiv:1107.1916 [astro-ph.CO]].

[30] R. C. Gilmore, R. S. Somerville, J. R. Primack and A. Dominguez, arXiv:1104.0671 [astro-ph.CO].

[31] R. K. Sheth and G. Tormen, Mon. Not. Roy. Astron. Soc. 308 (1999) 119 astro-ph/9901122.

[32] R. K. Sheth, H. J. Mo and G. Tormen, Mon. Not. Roy. Astron. Soc. 323 (2001) 1 astro-ph/9907024.

[33] J. F. Navarro, C. S. Frenk and S. D. M. White, Astrophys. J. 490 (1997) 493 astro-ph/9611107.

[34] G. L. Bryan and M. L. Norman, Astrophys. J. 495 (1998) 80 astro-ph/9710107.

[35] J. S. Bullock, T. S. Kolatt, Y. Sigad, R. S. Somerville, A. V. Kravtsov, A. A. Klypin, J. R. Primack and A. Dekel, Mon. Not. Roy. Astron. Soc. 321 (2001) 559 astro-ph/9908159.

[36] A. R. Duffy, J. Schaye, S. T. Kay and C. Dalla Vecchia, Mon. Not. Roy. Astron. Soc. 390 (2008) L64 arXiv:0804.2486 [astro-ph]].

[37] W. Hu and A. V. Kravtsov, Astrophys. J. 584 (2003) 702 astro-ph/0203169.

[38] A. M. Green, S. Hofmann and D. J. Schwarz, Mon. Not. Roy. Astron. Soc. 353 (2004) L23 astro-ph/0309621.

[39] A. M. Green, S. Hofmann and D. J. Schwarz, JCAP 0508 (2005) 003 astro-ph/0503387.

[40] J. Diemand, B. Moore and J. Stadel, Nature 433 (2005) 389 astro-ph/0501589.

[41] J. Diemand, M. Kuhlen and P. Madau, Astrophys. J. 649 (2006) 1 astro-ph/0603250.

[42] S. Profumo, K. Sigurdson and M. Kamionkowski, Phys. Rev. Lett. 97 (2006) 031301 astro-ph/0603373.

[43] A. A. Abdo et al. [Fermi-LAT Collaboration], Phys. Rev. Lett. 104 (2010) 101101 arXiv:1002.3603 [astro-ph.HE]].
[44] A. A. Abdo et al. [Fermi-LAT Collaboration], Astrophys. J. 720 (2010) 435 arXiv:1003.0895 [astro-ph.CO]].

[45] J. Han, C. S. Frenk, V. R. Eke, L. Gao, S. D. M. White, A. Boyarsky, D. Malyshev and O. Ruchayskiy, arXiv:1207.6749 [astro-ph.CO].

[46] B. D. Fields, V. Pavlidou and T. Prodanovic, Astrophys. J. 722 (2010) L199 arXiv:1003.3647 [astro-ph.CO]].

[47] R. Makiya, T. Totani and M. A. R. Kobayashi, Astrophys. J. 728 (2011) 158 arXiv:1005.1390 [astro-ph.HE]].

[48] B. C. Lacki, S. Horiuchi and J. F. Beacom, arXiv:1206.0772 [astro-ph.HE].

[49] T. Narumoto and T. Totani, Astrophys. J. 643 (2006) 81 astro-ph/0602178.

[50] Y. Inoue and T. Totani, Astrophys. J. 702 (2009) 523 [Erratum-ibid. $\mathbf{7 2 8}$ (2011) 73] arXiv:0810.3580 [astro$\mathrm{ph}]$.

[51] K. N. Abazajian, S. Blanchet and J. P. Harding, Phys. Rev. D 84 (2011) 103007 arXiv:1012.1247 [astroph.CO]].

[52] M. Ajello et al., Astrophys. J. 751 (2012) 108 arXiv:1110.3787 [astro-ph.CO]].

[53] V. Springel et al., Mon. Not. Roy. Astron. Soc. 391 (2008) 1685 arXiv:0809.0898 [astro-ph]].

[54] M. Ackermann et al. [Fermi-LAT Collaboration], Phys. Rev. Lett. 107 (2011) 241302 arXiv:1108.3546 [astroph.HE]].

[55] A. Geringer-Sameth and S. M. Koushiappas, Phys. Rev. Lett. 107 (2011) 241303 arXiv:1108.2914 [astro-ph.CO]].

[56] S. Ando and D. Nagai, JCAP 1207 (2012) 017 arXiv:1201.0753 [astro-ph.HE]]. 